\title{
DIAGNÓSTICO DE SAÚDE DE COMUNIDADE COMO ATIVIDADE DISCENTE DE ENFERMAGEM, UTILIZANDO O MÉTODO DE PROJETO
}

\author{
Maria Yvone Chaves Mauro*
}

\begin{abstract}
RESUMO - Trata o estudo de uma experiência discente utilizando uma técnica administrativa - MÉTODO DE PROJETO - adaptado à prática de enfermagem, em Saúde da Comunidade. $\mathrm{O}$ estudo foi realizado por alunos do último período do Curso de Graduação da Escola de Enfermagem Ana Nery da UFRJ. A experiência visava desenvolver com os estudantes um método ativo de ensino e aprendizagem, a fim de capacitá-los a realizar todas as fases do Diagnóstico de Saúde de uma comunidade.
\end{abstract}

\begin{abstract}
It is a study on a learning experience that utilizes an adminstrative techine - PROJECT METHOD - adapted to nursing practice, in Community Health. The study was carried out at "Escola de Enfermagem Ana Nery da UFRJ" (Ana Nery School of Nursing of the Federal University of Rio de Janeiro) by senior students in their last year of Graduation Course. The experience aimed at developing an active teaching/learning method with the students, so as to qualifity them for the performing of all phases of Health Diagnosis of a community.
\end{abstract}

\section{INTRODUC̣ÃO}

Trata-se de uma experiência discente utilizando uma técnica administrativa - MÉTODO DE PROJETO - adaptado à prática de enfermagem em Saúde da Comunidade. Teve como propósito a realização de um Diagnóstico de Saúde, sob a coordenação da autora, através da elaboração e implementação de projetos.

A experiência foi vivenciada por sete grupos de aproximadamente 60 alunos por grupo, do último período do Curso de Graduação em Enfermagem e Obstetrícia da Escola de Enfermagem Ana Neri da UFRJ. Pretendeu-se cumprir os objetivos da etapa corricular "O profissional de Enfermagem e a Saúde da Comunidade", Unidade Programática "A Saúde da Comunidade em Micro-Regiões", visando desenvolver com os estudantes um método ativo de ensino aprendizagem a fim de capacitá-los a: analisar o contexto sócioecônomico e sanitário onde se desenvolve a prática da enfermagem comunitária; identificar necessidades de saúde da população e recursos assistenciais disponíveis da área estudada e aplicar uma tecnologia administrativa como processo de resolução de problema na área da saúde.

Os estudantes realizaram todas as fases do diagnóstico, a saber: reconhecimento da área a ser trabalhada; abordagens com os líderes da comunidade; delimitação dos objetivos e metas; elaboração dos instrumentos e coleta de dados; apuração e interpretação dos dados através de conceitos, tabelas e gráficos; análise e discussão dos resultados com os líderes da comunidade; proposição de soluções e elaboração do Documento Final que constitui a Avaliação Diagnóstica da área trabalhada.

\section{FUNDAMENTAC̣̃̃O TEÓRICA}

Evocando a responsabilidade do professor e do aluno na execução de ações no campo da saúde da comunidade, a experiência teve seu respaldo na Metodologia de Projeto já estudada pela autora em sua tese apresentada ao concurso de Professor Titular (1982). Uni-

\footnotetext{
* Maria Yvone Chaves Mauro, Doutor, Docente Livre e Mestre (EEAN - UFRJ).

Professora Titular do Departamento de Enfermagem de Saúde Pública (EEAN - UFRJ).

Enfermeira Sanitarista - ENSP - MS.

Enfermeira de Saúde Comunitária no CMS da IRA-SMS-RJ.
} 
ca referência encontrada, aplicando esta tecnologia como estratégia de ensino-aprendizagem em enfermagem.

Trata-se de uma tecnologia administrativa tendo com base o MÉTODO DE PROJETO. 0 embasamento teórico tem respaldo nos conceitos de Projeto, conceito de Saúde da Comunidade e outros correlatos; no estudo de indicadores de saúde; na interpretação dos papéis do enfermeiro na comunidade, e no estudo dos problemas e necessidades sócio-econômicas e sanitárias de uma população, em relação aos recursos assistenciais disponiveis.

Considerando-se o Método de Projeto uma tecnologia apropriada à Saúde da Comunidade, JEQUIER (1981) esclarece que teconologia apropriada é o conjunto de técnicas que ecologicamente se adaptam bem ao meio ambiente local, em pequena escala, e que envolvem recursos naturais. Esta expressão é aplicável a uma ampla gama de técnica de baixo custo, destinadas concretamente à atenção das necessidades mais básicas das populaçôes. Neste último sentido, a tecnologia apropriada centra-se principalmente nos problemas próprios dos países em desenvolvimento e pode ser aplicada à saúde, à produção de alimentos, à nutrição, ao trabalho, etc...

0 mesmo autor cita, ainda, que grande parte do gasto e do trabalho empregado na tecnologia apropriada, se dedica à investigação e ao aperfeiçoamento de novos produtos. A maior dificuldade que tem enfrentado os defensores da tecnologia apropriada, é o desenvolvimento dos meios, os instrumentos de administração, as formas organizacionais, os incentivos financeiros, as estruturas legais e o conhecimento cultural que determinam o êxito e o fracasso da inovação. Sem dúvida, seria um erro crer que os problemas que terá de enfrentar a tecnologia apropriada sejam exclusivamente de ordem técnica. Devemos reconhecer que levar a saúde para todos, com ajuda da teconologia apropriada, não é apenas uma questão técnica, mas sim, uma questão política.

Segundo o Informe do Comitê de Peritos da Organização Mundial de Saúde (OMS) ENFERMERIA Y SALUD DE LA COMUNIDAD (1985), a assistência de enfermagem a uma comunidade, preconiza o reconhecimento desta através do levantamento de dados demográficos, econômicos, sociais e de saúde, tomando como base a relação existente entre a Biologia Humana, os Modos de Vida da Comunidade, o Meio Ambiente e o Sistema de Assistência Sanitária e relacionando as seguintes categorias de informaçôes: dados demográficos, características do meio, informações de caráter econômico, necessidades de saúde e utilização dos meios de assistência utilizados pela população. Tendo como proposta final a "assistência de Enfermagem à comunidade", entende-se que este conceito, segundo a OMS compreende a assistência à família, a identificação das necessidades gerais de saúde da comunida- de e a tarefa de conseguir a participação da comunidade na execução dos projetos relacionados com a saúde e o bem-estar da população; ajuda às comunidades a idẹntificar seus próprios problemas, a encontrar soluções e a fazer tudo que está ao seu alcance antes de recorrer a ajuda exterior.

LAURENTI e PASTORELO in PARETA (1976) apontam os indicadores que servem de parâmetro para avaliar os fatores que afetam a saúde da população e os critérios que indicam melhorias, considerando como mais significativos:

- o número de vida

- o nível sócio-econômico

- o nível de saúde e

- a prestação de serviçõs de saúde

Esses indicadores servem para indicar o grau de necessidades da comunidade e conseqüentemente orientam as medidas de enfermagem a serem tomadas em conjunto com as ações de outros profissionais.

Segundo JACOBSON citado por ARCHER (1977), a Enfermagem de Saúde da Comunidade, é uma disciplina erudita de serviços, cujo propósito é contribuir, de modo individual e em colaboração com os demais membros da equipe de saúde para a promoção do Nível Funcional Otimo (NFO) do cliente, da família e da comunidade mediante 0 ensino e a prestação de cuidados.

Alguns papéis a enfermeira de saúde da comunidade desempenha neste trabalho: de advogado, de colaborador e membro da equipe, de monitor, de coordenador e facilitador, de administrador de serviços, de educador e de investigador. JACOBSON considera que a Enfermeira de Saúde da Comunidade tem falhado por haver feito escassa investigação e não ter bases teóricas para exercer sua prática.

Durante muitos anos os educadores de enfermagem mantiveram a crença de que a investigação em enfermagem era prerrogativa de pós-graduação. Entretanto, é través dos dados qualitativos e quantitativos sobre um cliente ou uma população que se pode estabelecer um plano adequado de intervenção.

Segundo FREEMAN (1971) o Diagnóstico de Saúde Coletiva é a chave da prática da saúde social na medida em que os objetivos da saúde sejam compreendidos pela população e que a melhoria de sua qualidade está em relação direta com a prevenção e o controle das enfermidades e com os ef eitos das forças ecológicas e psicológicas sobre saúde, a população terá melhores condições de sobreviver no seu meio.

Assim sendo, o diagnóstico de saúde de uma comunidade, reflete não só o registro conjunto do estado de saúde de um grupo determinado de população, como também faz uma apreciação da situação dinâmica em que ditos grupos experimentam, adaptam e modificam todas as forças biológicas, físicas e sociais relacionadas com a saúde, assim como suas relações com as ações e reações destas forças entre si. 
A enfermeira de comunidade deve compreender o processo mediante o qual se obtém um diagnóstico de saúde coletiva, que são os mesmos em todos os níveis de complexidade de assitência, em que pese haver diferenças nos tipos de dados coletados para analisar, a saber:

a) estado de saúde da coletividade incluindo o nível de vulnerabilidade da população, assim como os fatores humanos e fatores ambientais intervenientes;

b) capacidade do sistema de saúde para resolver os problemas de saúde incluindo os recursos humanos e materiais;

c) as prioridades de ação social, as formas e as diretrizes segundo as quais são elaborados os programas de saúde, incluindo o sistema político local.

DUARTE citado por PARETA (1976) diz que o Dianóstico de Saúde de uma comunidade deverá ser feito atraveś do levantamento de todos os dados, permitindo não somente o reconhecimento dos problemas de saúde como, também, o seu dimensionamento, o que levará não somente a quantificação dos danos conhecidos, assim como a identificação das causas determinantes.

0 reconhecimento das causas leva ao estudo e seleção de possbilidades técnicas de combatê-las, seja através de recursos já existentes ou possíveis de serem obtidos.

As possibilidades de saúde de uma comunidade estão representadas pelo grau em que esta é capaz de resolver seus próprios problemas e necessidades, isto é, na medida em que seus recursos econômicos, institucionais e humanos são suficientes visando assegurar o bem-estar da população envolvida.

Um diagnóstico em termos de comunidade, não necessita ser completo porque um diagnóstico completo é quase impraticável. Entretanto o Diagnóstico deve ser útil e relacionado com o trabalho dos órgãos de saúde que servem aquela área. Desse modo, o diagnóstico pode ser dirigido para solucionar problemas mais significativos em relação aos recursos disponíveis ou para solucionar problemas indicados pela população quando questionada.

GIDEON (1979) reforça que o Diagnóstico de Saúde da Comunidade, avalia a comunidade como um todo e visa o alcance dos objetivos a seguir indicados:

- identificação dos problemas da comunidade;

- estabelecido de prioridades para o planejamento e desenvolvimento de problemas de assitência à saúde;

- proposição de soluções viáveis.

É uma experiência de aprendizado contínuo, tanto para o coordenador quanto para os participantes e para a própria comunidade, possibilitando

a) ajustar ou alterar programas já existentes visando alcançar o maior grau possível de eficácia; b) tornar a comunidade gradualmente consciente da situação existente;

c) fazer com que a comunidade atenda de acordo com o seu próprio rítmo, suas vantagens potenciais de mudanças, de atitudes, valores e comportamento.

Um Diagnóstico de Saúde de Comunidade, é mais ou menos semelhante ao diagnóstico clínico de um paciente havendo apenas as seguintes diferenças:

- enquanto o paciente procura o médico para ajudálo, a comunidade pode ou não estar consciente de seus problemas, e raramente pede ajuda, orientação ou assistência por sua própria iniciativa;

- a doença do paciente é uma condição da patologia geralmente exclusiva, enquanto os problemas da comunidade não podem ser tratados isoladamente porque são interligados a outros fatores.

GIDEON (1979), sugere as seguintes etapas para a elaboração do Diagnóstico de Comunidade, as quais tem semelhança com o diagnóstico clínico:

1. Levantamento bibliográfico (dados sobre a comunidade e as técnicas indicadas para realizar 0 trabalho);

2. Reconhecimento do campo;

3. Levantamento dos dados demográficos e específicos das condições da população para avaliar até que ponto o problema identificado afeta a população ou parte dela (ex: grupo de hipertenso), e avaliação dos recursos disponíveis para minorar os problemas (ex: serviços de controle de hipertensos);

4. Identificação do comportamento comunitário para localizar grupos contra ou favor das soluções propostas, levantar as dificuldades a serem superadas, encontrar soluções possíveis e viáveis, determinar adequação ou inadequação de outras entidades que atuam na área e definir a reorientação do sistema de saúde local;

5. Elaboração do documento final do Diagnóstico e o Plano Intervenção ou Programa, com alternativa de solução a curto, médio e longo prazos.

Usou-se a TÉCNICA DO PROJETO, denominada por GONÇALVES (1976) como METODOLOGIA DE PROJETOS que tem a capacidade de proporcionar o desenvolvimento do ensino-aprendizagem através de: esforço próprio do estudante (aprender fazendo); motivação do aluno para sua aprendizagem; uso de uma situação real de aprendizagem; globalização do ensino (percepção do todo para depois perceber as partes).

Além dos aspectos psicológicos de aprendizagem, tem-se como positivos os aspectos sociológicos: socialização do educando, integrando-se na vida social da comunidade; ensaios de auto-determinação ao tomar decisões junto aos líderes; trabalho em equipe, desenvolvendo espírito de solidariedade e cooperação.

Do ponto de vista administrativo, CUDIERMAN citado por MAURO (1982) define o PROJETO como "Sis- 
temas finitos, complexos, não homogêneos e não repetitivos considerando o prazo (período), o preço (orçamento), a performance (desempenho técnico) e o perigo (riscos) como seus termos de referência, de acordo com a política adotada. Este conceito é entendido como a regra dos CINCO Ps os quais são considerados como indicadores de êxito do Projeto".

RAYMOND LEON citado por MAURO (1982) completa a indicação de Projeto como técnica adminsitrativa definindo o Projeto como "a menor unidade de um plano ou programa, possível de identificação, implementação e avaliação independente, informando-se daí o Projeto a unidade básica, o que, sem tentativa de estabelecer rigidez hierárquica, induz a considerar o plano como um coletivo de programas, e este como coletivo de Projetos".

Os projetos são desenvolvidos para criar um produto final:
a) bens de consumo ou equipamentos;
b) instalações;
c) informaçẽos;
d) serviços

Na linha de serviços (letra d) é que se pretende utilizar o MÉTODO DE PROJETO como teconologia apropriada, adaptada ao serviço de Enfermagem à Comunidade.

\section{ESTRATÉGIAS DE EXECUC̣ÃO:}

O Diagnóstico de Saúde da Comunidade, apesar de ser iniciado por interesse didático também se adequa ao interesse administrativo da Região, visando a coincidir com a proposta de trabalho de COGESTÃO do CONASP (Ministério da Saúde, Estado, Município, Universidade e INAMPS) que opera no município, por meio da Comissão Interinstitucional Municipal de Saúde (CIMS) e esta interage nas regiões através de uma Comissão Executiva (CEAP). Esta por sua vez operacionaliza suas açẽos em Regiões de Saúde, designadas como GRUPO EXECUTIVO LOCAL (GEL), o qual corresponde a uma área de Planejamento do Município (API).

Fazem parte desta API sete Regiões Administrativas (RA) entre as quais está incluida a I RA, alvo do interesse do Diagnóstico aqui relatado.

Este processo de Ação Comunitária foi proposto pela COGESTÃO a fim de operacionalizar as Ações Integradas de Saúde (AIS), estratégia de cobertura de saúde adotada pelo Ministério de Saúde para todo o país.

$\mathrm{Na}$ área em que é relatada a experiência, o trabalho de Diagnóstico antecedeu à determinação oficial em 1986, uma vez que este tipo de trabalho já vem sendo feito em outras regiões desde o primeiro semestre de 1983 e na I RA, alvo deste relato, desde o primeiro semestre de 1985. A região possui aproximadamente 65 mil moradores localizados em duas sub-regiões; uma, mais tradicional, de condições sócio-econômica melhores,com população fixa; a outra relativamente agrega- da, mais recentemente, é composta de migrantes com um nível econômico e social baixo, e correspondente aproximadamente 35 mil moradores. Esta foi a área definida para o levantamento de dados na comunidade embora tenha sido dimensionados os recursos de toda a I RA. Esta Região é composta de nove pequenas comunidades favelas ou parques residenciais, todas elas com seus líderes conhecidos e participantes do Conselho Comunitário Participativo, o qual envolve também representantes of iciais dos órgãos locais da administração, da educação e da saúde. Embora os estudantes não tivessem tal vivência nesta área, a coordenadora desenvolve um bom relacinamento na região escolhida atuando como Enfermeira de Saúde Comunitária. Dessa vivência e participação, e de acordo com o interesse do líder da Saúde Pública Sanitarista e Diretor do Centro Municipal de Saúde, foi proposto a realização do Diagnóstico de Saúde, por etapas, utilizandose a participação dos estudantes. Tal proposta foi aceita pelos líderes das comunidades envolvidas.

Já havia sido feito semelhante trabalho em comunidades de outras regiões, com outras turmas, usandose várias estratégias, em busca de um modelo mais adequado, uma vez que a clientela de alunos do curso é de aproximadamente 60 alunos por período, número que dificulta o trabalho em grupo, com apenas um ou dois professores.

Foi definida a sub-área mais pobre da Região para ser questionada sobre a situação social, econômica e de saúde, a partir de levantamento domiciliar.

Feita a seleção, foram convidados os líderes das comunidades e os líderes de saúde interessados nos problemas de saúde da região incluindo-se o Diretor do Centro de Saúde, para acompanhar o desenvolvimento do trabalho e posteriormente sugerir questões na elaboração dos formulários para coleta. Alguns forneceram sugestões para a coleta de dados; visando o interesse de sua própria comunidade, outros forneceram o mapa da região; outros fizeram a divulgação do trabalho junto aos moradores e outros, ainda, participaram da visita de reconhecimento das localidades.

o Diretor do Centro de Saúde of ereceu o apoio legal e participou de todas as fases do processo, inclusive opinando no trabalho uma vez que os resultados obtidos seriam de grande interesse para o planejamento local.

O Diretor do Centro de Saúde of ereceu o apoio legal e participou de todas as fases do processo, inclusive opinando no trabalho uma vez que os resultados obtidos seriam de grande interesse para o planejamento local.

Alguns outros dirigentes de instituições como o do Serviço de Fisiologia e Pneumologia solicitaram incluir dados para identificar sintomas respiratórios. Os líderes de comunidades, de modo geral solicitaram investigar sobre a participação dos moradores na sua Associação de Bairro e a razão porque não participam. 
Houve a participação de um professor do Curso de Estatística da mesma Universidade o qual colaborou efetivamente da definição da amostra, na elaboração dos formulários de coleta e na apuração de dados que por duas vezes foi feita em computador com a participação dos alunos de pós-graduação em Estatística e do curso de Amostragem.

A participação efetiva dos estudantes de Enfermagem foi na Elaboração do Projeto de Diagnóstico, desenvolvimento do Projeto, Avaliação ao Término do Projeto, Apresentação dos resultados em Seminários, com a participação dos líderes de Saúde e das Comunidades envolvidas, com o objetivo de discutir os problemas encontrados e fazer encaminhamento das soluções propostas.

Foram elaborados um ROTEIRO SIMPLIFICADO PARA ELABORAÇÃO DE PROJETOS, já experimentado com mais de 70 Projetos de Enfermagem e um ROTEIRO PARA AVALIACূ̃̃O DOS RESULTADOS DO PROJETO INDICADORES DE SUCESSO NUM PROJETO, ambos já testados com resultado positivo apresentado em anexo.

A estratégia utilizada para a consecução do Diagnóstico de Saúde da Micro-Região (sub-área da I RA) foi dividir o trabalho em partes, operacionalizando os Projetos com um turma de alunos em cada semestre do ano letivo. Assim sendo já temos concluido: no semestre 1/85 - duas comunidades; semestre $2 / 85$ - três comunidades e semestre 1/86 - levantamento dos recursos institucionais de Saúde a fim de avaliar a oferta de serviços de saúde em relação as necessidades identificadas pelas comunidades.

Foram seguidas as seguintes etapas para 0 desenvolvimento do trabalho: Estudo da literatura disponível; Visita de reconhecimento à comunidade e contato com as lideranças locais; Mapeamento da região a ser trabalhada com localização de suas moradias ou instituições; Dimensionamento da amostra; Elaboração do projeto; Elaboração do formulário de coleta de dados; Treinamento dos entrevistadores (alunos) e divisão do trabalho de campo; Confirmação de datas para a coleta; Levantamento dos dados em campo; Tabulação e apuração dos dados; Elaboração do Relatório Final e Plano de Intervenção; Apresentação dos resultados em Seminários com vistas a elaboração do Plano de Intervenção; Aplicação do Plano de Intervenção com soluções em aberto (a serem resolvidas pela enfermagem ou encaminhadas a outros profissionais); Entrega do Projeto com o Relatório Final a quem couber (Professores, Autoridades e Comunidade).

\section{RESULTADOS}

Os projetos foram desenvolvidos em tempo hábil pelos próprios alunos com a participação dos professores, dos profissionais de saúde envolvidos e líderes das comunidades.
Todos os projetos são integrados entre si porque fazem parte do PROGRAMA que tem como objetivo o DIAGNÓSTICO DE SAÚDE DA MICRO-REGIÃO, de interesse do Centro de Saúde e da Coordenação Local da COGESTÃO, que aspira ter um Diagnóstico completo da API. Enquanto a I RA (qeu integra as 7 RAS da API) já dispõe de um Diagnóstico sendo concluído, a CEAP ainda está planejando uma etapa do Diagnóstico da API, começando com o levantamento dos recursos institucionais da área.

0 próprio Ministério em suas NORMAS e MANUAIS TÉCNICOS DE ORIENTAÇÃO PARA ORGANIZAÇÃO DE CENTROS DE SAÚDE: O PLANEJAMENTO LOCAL de 1985, define bases para elaboração do Diagnóstico de Saúde como parte do Planejamento no setor de Saúde, prática que a Escola de Enfermagem Ana Neri vem desenvolvendo desde 1982.

A prática de desenvolvimento de PROJETOS com Estudantes de Enfermagem vem sendo desenvolvida com resultados positivos há mais de 15 anos, documentado na Tese de Titular de MAURO (1982) e em outros trabalhos divulgados da mesma autora. (1981).

\section{CONCLUSÕES}

1. Sendo o Diagnóstico de Saúde um processo baseado na realidade da comunidade e dos serviços, os estudantes puderam identificar os problemas da clientela e aplicar seus conhecimentos na identificação das necessidades de Enfermagem, conforme preceito do Corrículo Integrado do Curso de Graduação em Enfermagem e Obstetrícia da EEAN-UFRJ.

2. É um processo de ensino-aprendizagem no qual o estudante interage com os serviços de saúde e com a comunidade, sendo também racional porque se fundamenta na análise das necessidades do serviço e atende as necessidades da demanda da clientela.

3. Os estudantes de enfermagem que participaram desta experiência, não chegaram a satisfação total porque, em todos os casos, o tempo (um semestre) não permitiu condições para solucionar todos os problemas identificados, os quais são encaminhados para os órgãos envolvidos com o Diagnóstico de Saúde, deixando um insatisfação dos discentes em relação à clientela.

4. A experiência com MÉTODO DE PROJETO atingiu um alto nível de satisfação em relação a avaliação dos resultados, concluindo-se ser um MÉTODO DE EXCELÊNCIA para aprendizagem em relação às várias atividades de enfermagem, incluindo o Diagnóstico de Saúde.

\section{SUGESTÕES}

1. Aos enfermeiros, que procurem integrar-se às equipes para elaboração de Diagnósticos de Saúde da Co- 
munidade nos processos de organização e reorganização de serviços, em qualquer nível (primário, secundário e terciário) especialmente nos trabalhos de enfermagem em comunidades.

2. Às Escolas de Enfermagem que desenvolvam o MÉTODO DE PROJETO para solução de problemas na área de enfermagem com a garantia de aquisição de uma visão global dos problemas de saúde, maturidade profissional e formação polivalente, devido às atividades diferenciadas.

\section{REFERÊNCIAS BIBLIOGRÁFICAS}

1. ARCHER, S.E. \& FLESHMAN, R. Enfermeira de Salud Comunitária. OPS/OMS, 1977.

2. BRASIL, Ministério da Saúde. Orientação para Organização de Centros de Saúde: O Planejamento Local. Brasília, Normas e Manuais Técnicos, 20, 1985.

3. FREEMAN, R.B. Enfermeira de Salud Pública. México, Nueva Ed. Interamericana. 1971.

4. GIDEON, H. Diagnóstico de Comunidade: Ponto de Partida para Programas de Saúde Comunitária/passos rumo a Saúde Comunitária. São Paulo, Ed. Paulinas, 1979.

5. GONÇALVES, R. Didática Geral. 8 ed., Rio de Janeiro, Liv. Freitas Bastos, S.A., 1976.

6. JEQUER, N. La tecnologia apropriada necessita "empuje" político. Foro Mundial de la Salud, 2(4):628-31, 1981.

7. MAURO, M.Y.C. Método de Projetos em Enfermagem de Saúde Pública. Rio de Janeiro, Escola de Enfermagem da UFRJ. Tese apresentada ao Concurso de Professor Titular. 1982.

8. Experiência Docente Assistencial: Efeitos sobre o rendimento dos alunos de Habilitação em Saúde Pública. Rio de Janeiro, 1981.

9. PARETA, J.M.M. \& D'ANDRETA Jr. A.R.M. Saúde da Comunidade, São Paulo, Ed. Mc Graw-Hill do Brasil Ltda, 1976.

\section{ANEXO I}

\section{ROTEIRO SIMPLIFICADO PARA ELABORA- ÇÃO DE PROJETO (aplicação em Enfermagem)}

\section{IDENTIFICAÇÃO DO PROJETO}

Órgão, Programa, Denominação, Datas previstas de Início e Término, localização e Gerência.

\section{JUSTIFICATIVA}

Breve relato sobre a origem do Projeto e a explicação de sua necessidade, com base em dados que permitam a delimitação do problema; justificar a ação proposta, na melhor alternativa de solução e os resultados esperados; indicar as interrelações e repercussões favoráveis.

\section{OBJETIVOS}

Geral: Explicitar de modo claro e preciso a finalidade do Projeto.

Específicos: Os resultados esperados, em termos operacionais orientados para a utilização dos recursos, obtenção dos resultados e retornos vantajosos.

\section{METAS}

Quantificação dos objetivos e sua localização no espaço geográfico e temporal. Define as etapas, as fases de execução, sua vinculação e orienta a liberação dos recursos.

\section{CUSTOS (GASTOS/ORÇAMENTO)}

Recursos necessários a execução do mesmo. Discrimina os custos das atividades, as fontes dos recursos, gastos previstos.

\section{ESTRATÉGIAS DE EXECUÇÃO}

Explicitar os procedimentos e os mecanismos através dos quais são desenvolvidas as ações necessárias para a consecução do Projeto. Órgãos envolvidos, cronologia das fases, responsabilidades definidas, coordenação, implementação, suporte, participação, interrelações.

\section{CRONOLOGIA DE EXECUÇÃO}

Prazos, Recursos e Produtos esperados.

\section{ACOMPANHAMENTO, CONTROLE E AVALIAÇÃO}

- Acompanhamento: Como fazer a verificação constante em termos de limites de tempo e espaço.

- Controle: Verificação da quantidade e qualidade.

- Avaliação: Estabelecer os critérios de como verificar o alcance dos resultados.

\section{RELATÓRIO FINAL}

Introdução

Desenvolvimento

Conclusões e Sugestões

\section{PLANO DE INTERVENÇÃO}

Propostas alternativas para a solução dos problemas. 